\title{
Developmental and behavioural characteristics of cri du chat syndrome
}

Room B55b, Faculty of Medicine, Medical School, University of Nottingham, Queen's Medical Centre, Nottingham NG7 2UH K M Cornish J Pigram

Correspondence to: Dr Cornish.

\author{
K M Cornish, J Pigram
}

\begin{abstract}
Developmental and behavioural characteristics were assessed in 27 children with cri du chat syndrome using the Society for the Study of Behavioural Phenotypes questionnaire, which gave information on prenatal and perinatal conditions, neurological problems, and developmental and behavioural difficulties. The findings suggest that the behavioural profile of children with cri du chat syndrome incorporates self injurious behaviour, repetitive movements, hypersensitivity to sound, clumsiness, and obsessive attachments to objects. In terms of a developmental profile, children with cri du chat syndrome were able to communicate their needs, socially interact with others, and have some degree of mobility.

(Arch Dis Child 1996;75:448-450)
\end{abstract}

Keywords: cri du chat syndrome, learning disability, chromosome disorder, behavioural characteristics.

Identifying the behavioural phenotype of individuals with genetically determined disorders has become a strong focus of research in recent years. Behavioural profiles have now been established for a number of syndromes including fragile X syndrome, ${ }^{1}$ Noonan's syndrome, ${ }^{2}$ Williams syndrome, ${ }^{3}$ and Turner's syndrome. ${ }^{4}$ These profiles can provide a useful indicator of the behavioural characteristics and problems associated with a given genetic disorder. The present study describes the behavioural phenotype of 27 children with cri du chat syndrome, a rare chromosome disorder that affects approximately one in 50000 live births. The syndrome is caused by a loss of chromosomal material from the critical region $5 p(5 p 12)$ and represents one of the most common deletion syndromes in humans. ${ }^{5}$ The main clinical characteristics include a distinctive, high pitched 'cat-like' cry ${ }^{6}$; a round or 'moon facies' with low set ears and asymmetrical facial contour ${ }^{5}$; and respiratory and cardiac abnormalities. $^{7}$

While identification of the clinical characteristics of cri du chat syndrome were first published over 30 years ago, few studies have sought to establish a cognitive and behavioural profile of this syndrome. Before 1980 the typical portrayal of the syndrome was one of profound mental retardation, limited verbal abilities, and slow psychomotor development. ${ }^{8}$ However, recent studies by Carlin and by Cornish have indicated that many children with cri $\mathrm{du}$ chat syndrome live at home, interact socially, and develop some language and motor skills. $^{910}$
The present study describes the developmental and behavioural profile of 27 children with cri du chat syndrome. The study forms part of a larger study examining cognitive and adaptive functioning in such children. As far as the authors are aware this is the first published study of the behavioural phenotype of cri du chat syndrome.

\section{Patients and methods}

PATIENTS

The participants were 27 children with cri du chat syndrome (mean chronological age: 8.3 years; age range 4.0-16.0 years; mean mental age: 4.8 years (age range $2.2-12.4$ years); there were 13 boys and 14 girls). Mental age was measured using the British picture vocabulary scale. ${ }^{11}$

Diagnosis had been established by chromosomal analyses and physical phenotype. Participants were recruited via the UK cri du chat syndrome association. All 27 of the families who were contacted participated in the study.

All the children in the sample were living in the community, none was living in institutional care; $16.7 \%$ attended a mainstream school with special needs provision; $37.5 \%$ attended a school for mild-moderate learning disabilities; and $45.8 \%$ attended a school for severe learning disabilities.

\section{QUESTIONNAIRE}

The Society for the Study of Behavioural Phenotypes questionnaire has been developed for the purpose of obtaining cross syndrome data and comprises four sections ${ }^{12}$ :

(1) Prenatal and perinatal conditions

(2) Neurological problems, specifically epilepsy

(3) Developmental assessment, including vision, hearing, mobility, dexterity, feeding, continence, dressing, hygiene, and communication

(4) Behavioural domains, including feeding and drinking, sleep, social behaviour, movement activities, difficulties with language, unusual movements and interests, aggressive behaviour, and anxiety and mood.

All 27 families were questioned during a semistructured interview when their child participated in an extensive neuropsychological study carried out by the authors.

\section{Results}

PRENATAL AND PERINATAL CONDITIONS

Of the 27 mothers, $10(37.0 \%)$ reported the presence of an abnormality during their pregnancy; $12(44.4 \%)$ reported that their 
Table 1 Summary of the developmental characteristics of children with cri du chat syndrome $(n=27)$

\begin{tabular}{lc}
\hline Assessment & No (\%) \\
\hline Vision/hearing & \\
Wears glasses & $4(14.8)$ \\
Wears a hearing aid & 0 \\
Mobility & $23(85.2)$ \\
Can sit without support & $5(18.5)$ \\
Walks steadily and normally & $10(37.0)$ \\
Walks, but not as steadily as other children & $7(26.0)$ \\
Walks, but activity limited & $5(18.5)$ \\
No independent walking & \\
Dexterity & $25(92.6)$ \\
Gross hand movements & $18(65.4)$ \\
Fine hand movements & \\
Feeding & \\
Feeds him/herself: & $1(3.7)$ \\
Not at all & $19(70.4)$ \\
Finger feeds & $5(18.5)$ \\
Spoon feeds & $2(7.4)$ \\
Uses knife and fork & $19(70.4)$ \\
Drinks from an ordinary cup & \\
Continence and hygiene & $7(25.9)$ \\
Toilet trained & $8(29.6)$ \\
Washes self totally & \\
Dressing & \\
Ability to dress him/herself: & \\
Completely & \\
With little help & \\
A lot of help & $13(29.6)$ \\
Not at all & $1(18.5)$ \\
Ability to comunicate & \\
How he/she makes needs known: & \\
Does not indicate needs & \\
Uses non-verbal method & \\
Uses formal sign or symbol system & $(18.4)$ \\
Uses speech & \\
& \\
& \\
&
\end{tabular}

baby had had difficulties during the delivery; and, over half of the children in the sample 14 $(51.8 \%)$ needed special care facilities directly after the birth.

NEUROLOGICAL PROBLEMS

None of parents in the sample reported that their child had had fits or epilepsy.

\section{DEVELOPMENTAL PROFILE}

Table 1 provides a summary of the developmental profile.

Abnormal vision was present in only four $(14.8 \%)$ children and there was no reported incidence of abnormal hearing. While the majority of all children in the sample had some degree of mobility, in that $23(85.2 \%)$ could sit without support, the ability to walk steadily and normally was severely impaired with only five $(18.5 \%)$ children able to walk competently. While gross motor skills (for example, waving) were achieved in $25(92.6 \%)$ of the children, only $18(65.4 \%)$ were competent in fine motor skills (that is, holding a pencil). Twenty six (96.2\%) children could feed themselves to varying degrees and $19(70.4 \%)$ could drink from an ordinary cup. However, fewer than a third of children were toilet trained (seven; $25.9 \%$ ) and only eight $(29.6 \%)$ were able to wash and dress themselves completely at the time of the assessment. The ability to use speech to communicate needs was evident in fewer than a third (seven; 25.9\%) of the children, although $15(55.5 \%)$ were able to communicate their needs using either nonverbal methods, $13(48.1 \%)$, or formal signs, two $(7.4 \%)$. Five $(18.5 \%)$ however were unable to communicate their needs using any of the above methods.
Table 2 Summary of the behavioural characteristics of children with cri du chat syndrome $(n=27)$

\begin{tabular}{|c|c|}
\hline Assessment & No (\%) \\
\hline \multicolumn{2}{|l|}{ Feeding } \\
\hline Normal intake of food & $19(70.4)$ \\
\hline Eating less than normal & $3(11.1)$ \\
\hline Eating more than normal & $5(18.5)$ \\
\hline Pica & $11(40.7)$ \\
\hline Faddy eating & $3(11.1)$ \\
\hline \multicolumn{2}{|l|}{ Sleep } \\
\hline Regular sleeping pattern & $20(74.1)$ \\
\hline \multicolumn{2}{|l|}{ Social behaviour } \\
\hline Normal interest in others & $10(37.0)$ \\
\hline Too friendly with strangers & $13(48.1)$ \\
\hline Unusual eye contact & $12(44.4)$ \\
\hline Facial grimacing & $9(33.3)$ \\
\hline \multicolumn{2}{|l|}{ Activity level } \\
\hline Underactive & $6(22.2)$ \\
\hline Normally active & $14(51.8)$ \\
\hline Overactive & $2(7.4)$ \\
\hline Clumsy & $19(70.3)$ \\
\hline Creates chaos aimlessly & $13(48.1)$ \\
\hline \multicolumn{2}{|l|}{ Communication } \\
\hline Echolalia & $14(51.8)$ \\
\hline \multicolumn{2}{|l|}{ Unusual movement and interests } \\
\hline Repetitive movements & $19(70.3)$ \\
\hline Peculiar object attachment & $14(51.8)$ \\
\hline Unusual reaction to light/sound/smell & $22(81.4)$ \\
\hline \multicolumn{2}{|l|}{ Aggressive/self injurious behaviour } \\
\hline \multicolumn{2}{|l|}{ Physically aggressive towards: } \\
\hline Family members & 9 (33.3) \\
\hline Non-family members & $5(18.5)$ \\
\hline Self injurious behaviour & $19(70.3)$ \\
\hline Stubborn & $16(59.2)$ \\
\hline Deliberately destroys things & 9 (33.3) \\
\hline \multicolumn{2}{|l|}{ Anxiety/mood } \\
\hline Excessively happy & $18(66.6)$ \\
\hline Excessively unhappy & $4(14.8)$ \\
\hline Often fearful & $4(14.8)$ \\
\hline Irritable & $10(37.0)$ \\
\hline Serious temper tantrums & $9(33.3)$ \\
\hline
\end{tabular}

BEHAVIOURAL PROFILE

Table 2 provides a summary of the behavioural profile.

There was one characteristic that occurred in more than $75 \%$ of the group and nine characteristics that occurred in more than $50 \%$ of the group: hypersensitivity to sensory stimuli $(81.4 \%)$, self injurious behaviour $(70.3 \%)$, repetitive movements $(70.3 \%)$, clumsy $(70.3 \%)$, excessively happy $(66.6 \%)$, stubborn $(59.2 \%)$, object attachments $(51.8 \%)$, echolalia $(51.8 \%)$, and normally active $(51.8 \%)$.

\section{Discussion}

The data presented here outline a profile of developmental and behavioural characteristics in children with cri du chat syndrome.

\section{DEVELOPMENTAL CHARACTERISTICS}

In terms of development, the main weaknesses involved those skills that required mobility, dexterity, and verbal communication. This finding was not unexpected and confirms that reported in earlier studies. ${ }^{7}$ However, examination of table 1 reveals that far from being totally immobile, maladroit and noncommunicative, the majority of children had acquired some degree of mobility, dexterity, and communication skill. For example, gross hand movements, in contrast to fine hand movements, were relatively unimpaired indicating an ability to use the hands for such tasks as waving or catching a rolling ball, if not for tasks that involve holding a pencil or putting pegs into holes. In addition, lack of speech did not appear to impede communication with 
more than two thirds of the children able to communicate their needs using non-verbal methods. However, relatively few children used formal sign language, which is suprising given recent evidence to indicate that over $50 \%$ of children with cri du chat syndrome were able to use sign language to communicate basic needs. ${ }^{9}$ Lack of training in formal sign language may have contributed to its low usage in the present study. Early stimulation and introduction to sign language are therefore crucial if more effective communication is to be attained.

\section{BEHAVIOURAL CHARACTERISTICS}

The behavioural profile for cri du chat syndrome appears to be one of self injurious behaviour, repetitive movements, obsessive attachment to objects, hypersensitivity to sensory stimuli, stubbornness, and clumsiness. Notably few children showed autistic characteristics (for example, gaze avoidance, social isolation), feeding or sleep problems, or mood disorders. What is particularly surprising, however, is the relatively low incidence of hyperactivity reported in the present study. This contrasts with an earlier finding by Wilkins et al which found that hyperactivity and restlessness were major problems in almost half of the 53 children assessed in their study. ${ }^{13}$

The extent to which these characteristics are specific to cri du chat syndrome or shared with other syndrome groups is difficult to measure without first ensuring that all samples are matched by intellectual level, sex, age, social class, etc. However, in comparison to other learning disabled groups, the incidence of repetitive movements and self injurious behaviours in cri du chat syndrome appears to be higher than the incidence reported in, for example, Down's syndrome (K Cornish, J Pigram, unpublished) or fragile X syndrome, ${ }^{1}$ but is comparable with the incidence reported in disorders such as Lesch-Nyhan syndrome. ${ }^{14}$

The high rate of hyperacusis reported in children with cri du chat syndrome is also a behaviour common to Williams syndrome, with a recent survey by Udwin revealing an incidence of over $90 \% .^{3}$ As in Williams syndrome, children with cri du chat syndrome become distressed at a wide range of sounds, including sudden noises made from aeroplanes, lawn mowers, a balloon bursting, thunder, etc. As yet, there is little evidence of specific auditory abnormalities to explain this hypersensitivity in cri du chat or Williams syndromes. However, early intervention to try and alleviate the distress caused by this condition is essential if the individual is to adjust into adulthood.

The high rate of clumsiness reported in children with cri du chat syndrome is hardly surprising given the restrictions imposed upon them by their lack of mobility and is prevalent in most syndromes where motor delay is a dominant feature (for example, Lesch-Nyhan syndrome). In contrast, an obsessional attachment to objects, which was present in over half of the children with cri du chat syndrome, is comparatively rare in other syndrome groups. The exceptions to this are Lowe's syndrome whose profile also includes repetitive movements and self injurious behaviour (although not hyperacusis) (Z Dolinsky, paper presented at Behavioural Phenotype Study Group Symposium, Welshpool, November 1990) and Williams syndrome whose profile also includes hyperacusis (but not repetitive movements or self injurious behaviour). ${ }^{15}$ The extent to which these obsessions persist into adulthood is not known but the effects in childhood are such that they place significant restrictions upon emotional and cognitive development.

While the present study has outlined a number of behavioural problems associated with cri du chat syndrome there are still many aspects of behaviour that are not as severely impaired and these need to be strengthened and encouraged by parents and professionals. The major limitation appears to be in activities and behaviours that require motor skill and it may well be the high incidence of some of the behavioural problems in this syndrome results from its physical constraints.

Our study has highlighted some of the developmental and behavioural aspects of cri du chat syndrome. In particular it has demonstrated the potential of children with this syndrome to develop and maintain important skills including ability to communicate needs, to socially interact with others, and to develop some degree of mobility. The extent to which these skills continue to develop in adulthood is not yet known, although considering the severity of the difficulties it is unlikely that adults with the syndrome will be able to live independently. However, this more realistic and optimistic portrayal of the syndrome should now enable parents and professionals to deal more effectively with the implications of a diagnosis of cri du chat syndrome in their child.

1 Dykens EM, Hodapp RM, Leckman JF. Behaviour and development in fragile- $X$ syndrome. London: Sage Publications, 1994.

2 Wood A, Massarano A, Super M, Harrington R. Behavoural aspects and psychiatric findings in Noonan's syndrome. Arch Dis Child 1995;72:153-5.

3 Udwin O. A survey of adults with Williams syndrome and idiopathic infantile hypercalcaemia. Dev Med Child Neurol 1990;32:129-41.

4 Rovet JF. The psychoeducational characteristics of children with Turner's syndrome. Fournal of Learning Disabilities with Turner's

5 Niebuhr E. Cytologic observations in 35 individuals with 5p-karyotype. Hum Genet 1978;42:143-56.

6 Lejuene J, Lafourcade J, Berger R, et al. Trois cas de deletion partielle du 13ras court d'un chromosome 5. C R Acad Sci (Paris) 1963;257:3098-102

7 Wilkins LE, Brown JA, Nance W, Wolf B. Clinical heterogeneity in 80 home-reared children with the cri-du-chat syndrome. F Pediatr 1983;102:528-33.

8 Silber DL, Engle E, Merrill RE, So called 'cri du chat syndrome'. American fournal of Mental Deficiency 1966;71 486-91.

9 Carlin ME. The improved prognosis in cri du chat (5p-) syndrome. In: Fraser WI, ed. Key issues in mental retardation research. London: Routledge, 1990.

10 Cornish $\mathrm{K}$. The neuropsychological profile of cri du chat syndrome without significant learning disability: a case syndrome without significant learning disa

11 Dunn L, Whetton C, Pintilie D. British picture vocabulary scale. Windsor: NFER, 1982.

12 O'Brien G. Behavioural phenotypes and their measurement. Dev Med Child Neurol 1992;34:365-7.

13 Wilkins LE, Brown JA, Wolf B. Psychomotor development in 65 home-reared children with cri du chat syndrome. $f$ Pediatr 1980;97:401-5.

14 Anderson LT, Ernst $M$. Self-injury in Lesch-Nyhan syndrome. $\mathcal{F}$ Autism Dev Disord 1994;24:67-81.

15 Kenworthy L, Park T, Charnas LR. Cognitive and behavioural profile of the oculocerebrorenal syndrome of Lowe. Am $\mathcal{F}$ Med Genet 1993;46:297-303. 